\title{
Gastric Malignancy Causing E. Coli Infection of a Frozen Elephant Trunk Stent Graft and the use of Transthoracic Composite muscle Flaps for Graft Salvage
}

\author{
Hui Chai FONG ${ }^{1}$, Victor Tar Toong CHAO ${ }^{2}$, Jason Hongting LEUNG ${ }^{2}$ and Bien-Keem TAN ${ }^{1 *}$
}

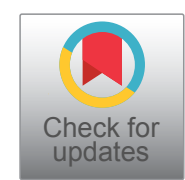

${ }^{1}$ Department of Plastic Surgery, Singapore General Hospital, Singapore

${ }^{2}$ Department of Cardiothoracic Surgery, National Heart Centre, Singapore

\section{Introduction}

Transthoracic muscle transposition is a useful technique for the treatment of intrathoracic air leak or empyema [1]. We describe an unusual case of gastric malignancy presenting as Escherichia Coli infection of a frozen elephant trunk stent graft, with associated empyema that was successfully salvaged with decortication and aneurysm resection. Intrathoracic transposition of a combined latissimus dorsi / serratus anterior flap was used for salvage of the stent graft.

\section{Case Report}

A 70-year-old gentleman with a medical history of type 2 diabetes mellitus, ischaemic heart disease, peripheral vascular disease and distal aortic arch aneurysm status post ascending and aortic arch replacement and frozen elephant trunk procedure using the Thoraflex ${ }^{\mathrm{TM}}$ Hybrid (Terumo, Japan) in 2016 presented with a 2-week history of fever and dyspnea. Auscultation revealed reduced breath sounds on the left, and chest x-ray showed whiteout of the left lung. Computed tomographic imaging of the aneurysm showed an increase in size of the previously excluded aneurysm, resulting in compression of the left main bronchus and causing left lung collapse. Decortication of the empyema and drainage of the aneurysm sac resulted in exposure of the aortic stent graft to the pleural space, necessitating the intrathoracic transposition of the latissimus dorsi (LD) muscle for obliteration of the aneurysm sac and coverage of the stent graft (Figure 1).

The latissimus dorsi and serratus anterior (SA) muscles were dissected and raised through an extended posterolateral thoracotomy incision. The LD muscle was raised with the SA muscle, sparing the distal attachments of the SA to the scapular tip. The long thoracic nerve was preserved. $A 4^{\text {th }} / 5^{\text {th }}$ thoracotomy allowed for decortication of the left lung and aneurysm resection, resulting in exposure of the aortic stent graft.

An accessory $3^{\text {rd }}$ thoracotomy was created, and the LD transposed intrathoracically, obliterating the space around the aortic arch and excluding it from the pleural space. Closed suction drainage was used to maintain a net negative pressure between the stent graft and flap, creating a seal around the latissimus dorsi muscle flap. The upper slips of the SA were transposed to seal the accessory thoracotomy defect, reconstituting the seal of the thoracic cavity and preventing air leak into the subcutaneous space. The donor site was closed with progressive tension sutures over suction drainage. 2 weeks later, an additional stent graft was successfully deployed via the transapical approach (transfemoral access was unsuccessful due to severe peripheral vascular disease) to extend the existing frozen elephant trunk down the descending thoracic aorta in order to provide a margin of safety with regards to the distal seal zone.

Intraoperative tissue cultures returned positive for Extended Spectrum Beta-Lactamase (ESBL) Escherichia Coli, prompting an investigation for a gastrointestinal source of bacterial translocation. Oesophagogastroduodenoscopy was performed, at which time a malignant looking antral ulcer was detected and biopsied, confirming moderately differentiated gastric antral adenocarcinoma. Staging scans were negative for metastatic disease and a joint decision was made with the patient for staged management of the gastric cancer after stabilization of his cardiothoracic issues.

6 weeks later, the patient underwent open subtotal gastrectomy and D2 lymphadenectomy successfully. The pathological staging was T1bN1M0 and the patient has been on routine surveillance post-operatively. The patient has remained well at 8 months with full shoulder range of motion and no endoleak on CT aortography (Figure 2).

*Corresponding author: Bien-Keem Tan, Department of Plastic Surgery, Singapore General Hospital, Outram Road, 169608, Singapore

Accepted: July 21, 2021

Published online: July 23, 2021

Citation: FONG HC, CHAO VTT, LEUNG JH, et al. (2021) Gastric Malignancy Causing E. Coli Infection of a Frozen Elephant Trunk Stent Graft and the use of Transthoracic Composite muscle Flaps for Graft Salvage. J Cardiothorac Surg Ther 5(1):95-97 
Citation: FONG HC, CHAO VTT, LEUNG JH, et al. (2021) Gastric Malignancy Causing E. Coli Infection of a Frozen Elephant Trunk Stent Graft and the use of Transthoracic Composite muscle Flaps for Graft Salvage. J Cardiothorac Surg Ther 5(1):95-97

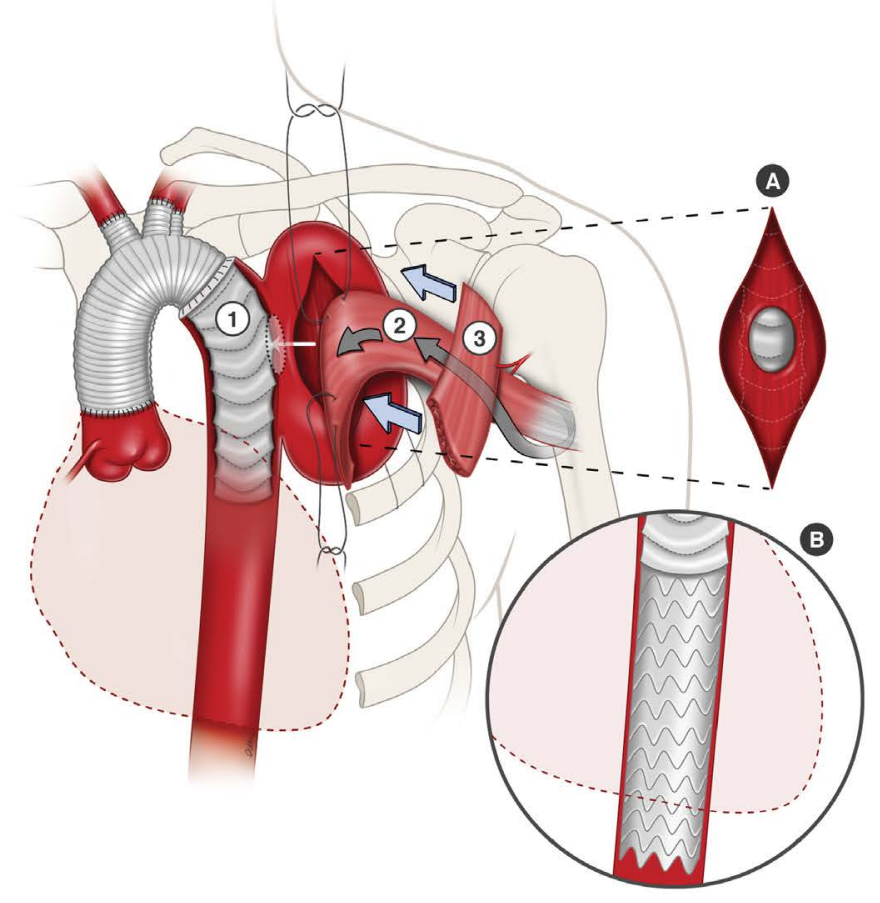
(1) Frozen elephant trunk graft
A Aortic defect, intra-sac view
(2) Latissimus dorsi muscle flap
B TEVAR stent deployed later
(3) Serratus anterior muscle flap

Figure 1: Illustration of graft salvage with intra-thoracic transposition of the latissimus dorsi muscle; A) use of the serratus anterior 3) to reduce air leak from the pleural cavity.

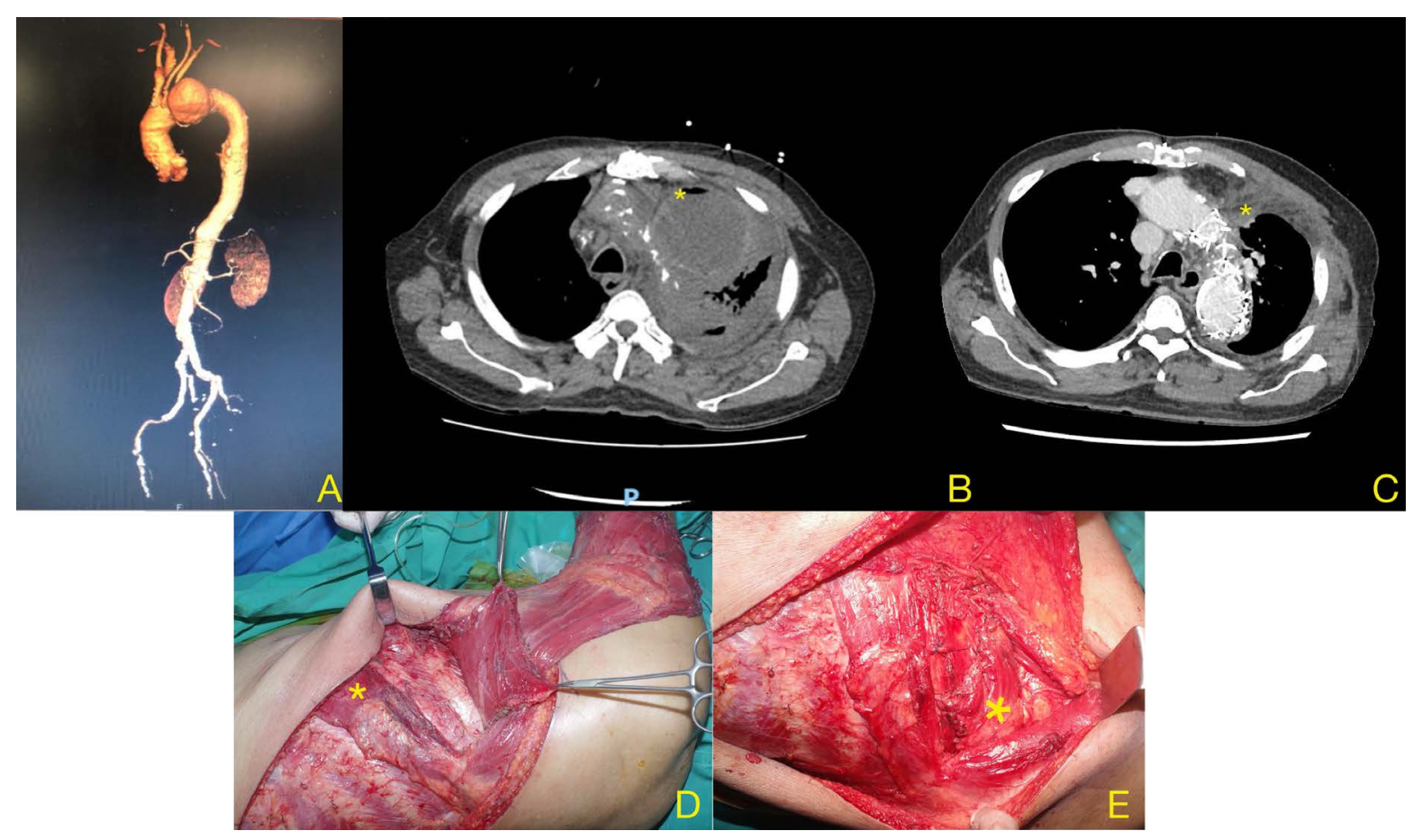

Figure 2: A) 3D CT scan detailing the location of the previously stented aortic aneurysm; B) The axial cut shows an air-fluid level in the pus-filled cavity; C) Post-operative axial CT scan showing the latissimus dorsi muscle's coverage of the aortic stent $\left({ }^{*}\right)$; D) The middle slips of the Serratus anterior muscle spanning the third-sixth ribs were mobilised in chimeric fashion based on the serratus branch of the thoracodorsal pedicle. The lower slips $(*)$ were left intact to prevent scapular winging; E) Utility of the serratus muscle flap $(*)$ in repairing the third rib defect through which the latissimus dorsi muscle was passed. 
Citation: FONG HC, CHAO VTT, LEUNG JH, et al. (2021) Gastric Malignancy Causing E. Coli Infection of a Frozen Elephant Trunk Stent Graft and the use of Transthoracic Composite muscle Flaps for Graft Salvage. J Cardiothorac Surg Ther 5(1):95-97

\section{Discussion}

The treatment of an infected thoracic aortic graft typically involves surgical debridement, graft removal and redo aortic replacement. However, revision arch replacement can be challenging in the setting of infection due to the presence of adhesions, indistinct surgical planes, and friable tissues. Furthermore, arch replacement requires cardiopulmonary bypass and circulatory arrest. An alternative strategy [2] of debridement, graft preservation with muscle flap transposition was employed in this patient. Intra-thoracic muscle transposition is useful for obliteration of postresection spaces, reinforcement of repaired broncho-pleural fistulas and coverage of exposed vessels $[3,4]$. While other options such as omentum, intercostal muscles or pericardial flaps have been used, the transposed latissimus dorsi muscle flap is advantageous due to its large size, thickness, vascularity and mechanical strength. The robust striated muscle is especially suited for the reinforcement of vascular structures and bronchial stumps as they are able to resist mechanical stresses during deep inspiration and coughing.

The use of the serratus anterior in combination with the latissimus dorsi expands on this technique by providing a secondary flap to close the thoracic window, compartmentalizing the pleural cavity from the large subcutaneous space that was created by the harvest of the latissimus dorsi muscle. This reduces air leak into the subcutaneous space from the pleural cavity, allowing the skin flaps to bed down, preventing seroma and wound dehiscence. It also confines the spread of any recurrent infection within the thoracic cavity, ensuring that pleural exudates drain through chest drains rather than into the subcutaneous space.

Anaccessory $2^{\text {nd }}$ or $3^{\text {rd }}$ thoracotomy, in the mid-axillary line, is useful in maximizing reach into the thoracic cavity. We prefer not to pass the muscle through an intercostal space as the pedicle might be compressed. Muscle adherence to the wound bed is optimized with the use of direct suture or closed suction drainage. Scapular winging is prevented by preserving the lower slips of the serratus anterior, stabilizing the scapular tip. In a previous series utilizing this technique for treatment of air leak, all patients $(n=5)$ had preserved shoulder range of motion and no scapular winging [1].

The haematogenous inoculation and infection of a previously excluded aortic aneurysm with ESBL E. Coli is an unusual manifestation of gastrointestinal malignancy. Our patient presented with several life-threatening pathologies; this complex case highlights the need for a high index of suspicion and multi-specialty consultation for the effective management of each condition. Intrathoracic muscle transposition was a critical component of the salvage of the aortic stent graft and prevention of life threatening haemorrhage. The serratus anterior flap is a useful adjunct to prevent subcutaneous air leak and to contain recurrent infection.

\section{Conflicts of Interest and Sources of Funding}

None declared.

\section{References}

1. Woo E, Tan BK, Lim CH (2009) Treatment of recalcitrant air leaks: The combined latissimus dorsi-serratus anterior flap. Annals of plastic surgery 63: 188-192.

2. Umminger J, Krueger $H$, Beckmann E, et al (2016) Management of early graft infections in the ascending aorta and aortic arch: a comparison between graft replacement and graft preservation techniques. Eur J Cardiothorac Surg 50: 660-667.

3. Arnold PG, Pairolero PC (1990) Intrathoracic muscle flaps. An account of their use in the management of 100 consecutive patients. Annals of surgery 211: 656.

4. Arnold PG, Pairolero PC (1988) Intrathoracic muscle flaps in the surgical management of life-threatening hemorrhage from the heart and great vessels. Plast Reconstr Surg 81:831-837.

DOI: $10.36959 / 582 / 434$

Copyright: (C) 2021 FONG HC. This is an open-access article distributed under the terms of the Creative Commons Attribution License, which permits unrestricted use, distribution, and reproduction in any medium, provided the original author and source are credited. 